\title{
Pittinsky Todd L (2019) Science, Technology, and Society: New Perspectives and Directions. Cambridge: Cambridge University Press. 270 pages. ISBN 978-1-316-61689-5
}

\author{
Josie Coburn \\ josie.coburn@sussex.ac.uk
}

This book aims at bringing together essays on the interplay between science, technology and society (STS). The essays within the book span a diversity of topics, including educational programs, scientific communities, technological decisionmaking, legal regulation, the role of users and nonusers in technological development, and genetic engineering and society. The book draws from a wide range of theoretical perspectives, including psychology, sociology, organisational studies and economics. Overall, it shows a stronger emphasis on technology than on science, although some chapters focus on science or on both, and the two cannot always be disentangled.

Several essays in this book situate current and new directions in STS in relation to the origins of the discipline, providing detailed historical accounts of how prominent themes have developed over time and how they might continue to evolve. Readers who would like to familiarise themselves with some of the more established topics within STS, for instance, may wish to start by looking at these.

Chapter 6 on the social shaping of technology (SST) provides an excellent example of this format. From the 1980s onwards SST has sought to answer questions about how technologies are shaped by socioeconomic, political and material factors. In this chapter, Williams traces the origins of the field and the early studies which constituted it; compares SST with the neighbouring field of actor-network-theory (ANT), in which both humans and objects are conceptualised as actors; surveys the move from SST Mark I to SST Mark II, in which theories of the relationships between technology and society became more complex; and reviews more recent conceptual and methodological directions, which provide evolutionary understandings and combine attention to local context and broader processes of sociotechnical change.

Collins and Evans offer a similarly organised account of the evolution of theories on the role of expertise in technological decision-making in the public domain, another enduring theme within STS. In chapter 4, the authors argue that we have reached a "Third Wave of science studies", which emphasises the need to combine specialist expertise and democracy to make the "best" decisions. Prior theories tended to either promote an excessive reliance on science (first wave) or a suspicion of expertise leading to overreliance on lay knowledges (second wave). Types of expertise are then categorised on the basis of tacit knowledge and socialisation.

In chapter 3, Godin provides a rich history of how different disciplines have theorised innovation over time, moving from linear models towards system models. In a context of economic, social, organisational and technological change, questions arise about how this change can be accelerated or directed towards desired goals. Historically, different theories on technological 
innovation have shared a representation of innovation as a process, and yet Godin argues that more recent theories on innovation as a system, while they still typically address innovation as a process, they tend to emphasise structure over time.

Chapter 5 addresses the multiple relationships between emerging technologies and regulation. According to Brownsword, the most important feature of this regulatory environment is that regulators should maintain the preconditions for human social existence itself. This involves upholding conditions for human existence, such as living within planetary boundaries; human agency, allowing humans to act in ways which are interactive and purposeful rather than merely defensive; and moral agency, to ensure that people have the freedom to choose to do the right thing and not to be compelled to do it via technologies.

Some chapters provide cross-cutting analyses by surveying the field through a particular lens, such as chapter 2, which provides a tour of prominent topics within STS through the lens of comparison. In this chapter, Horn argues that the so called "comparative method" actually covers a wide variety of different projects, but many of these projects have in one way or another been about "what science and technology have in common with other forms of human knowledge and human practice" (p. 52) and what is different, and that these are, at their heart, questions about culture. Comparative studies of science and technology can foreground discontinuities rather than continuity; different ways of knowing beyond the scientific method and rationality; and the roles played by cultural differences.

Simonton's chapter 8 presents a similarly extensive tour of STS theories through the lens of scientific communities. Within scientific communities, scientists typically reach a strong consensus on the questions, theories, methods and techniques that are considered relevant to the community, but research has shown that this consensus is socially constructed. Scientific communities are highly stratified with a small number of elite scientists, and early successes and failures in science cause large differences in the scientific productivity and status of individual scientists. More problematically, there is evidence of direct biases such as gender bias influencing scientific career trajectories. This chapter shows that scientists are embedded in rich social networks, and how this ultimately influences the nature of science.

In chapter 7, Oudshoorn surveys the role of users and non-users in relation to technological development. She shows that users play an active role, from design to implementation to use, and thus act as agents of sociotechnical change in a variety of ways. Additionally, technologies inside the body such as pacemakers have highlighted new forms of vulnerability in relationships between users and technologies because they are irreversible and the risks involved are unavoidable. Historically, non-users have been conceptualised in negative terms, such as laggards. However, non-users can also be rejectors or resistors, expelled or excluded, thus expanding categories of use and non-use. Furthermore, recent research has focused on "the blurry space" between the two.

In chapter 9, Barnes et al focus on the relationship between genetic engineering and society, conceptualising them as coproduced. Genetic engineering can also be described as a technoscience, blurring the boundaries between knowledge production and the production of products. Furthermore, a new technoscience typically emerges in an 'institutional void', and in this context science and technology can "overflow" the boundaries of institutions and laws. Public engagement initiatives have emerged, which open up spaces for the public to participate in the coproduction and democratisation of genetic engineering and governance.

In chapter 10, Mazei connects two streams of research, on sex differences, and on how technology shapes society and individual behaviours, to explore how technology can either enable or reduce sex differences, depending on the context. The chapter also proposes concrete actions to mitigate these effects, such as investing in technologies which facilitate non-traditional divisions of labour between the sexes; portraying women and men in a more balanced way in the media; and standardising workplace procedures such as hiring and promoting employees so that sex is ruled out as an influence. 
In the first essay of the book, Granger Morgan and Sicker write about the importance of truly combining technology and public policy education; the difficulty of maintaining a balance between the two; courses which do combine them; the relationship between this kind of education and policy analysis; and the impacts that an education which truly combines technology and public policy can achieve.

Pittinsky's final chapter provides a discussion of what 'technology for society' would look like, acknowledging that society is diverse and multilayered and so reaching consensus is not easy, but nevertheless arguing that there is a need to better understand how technologies could serve the greater good by promoting one or more of the following: good relationships; economic and material well-being; a health natural and built environment; health; peace and security; culture and leisure; spirituality, religion and ethics; good education; and good governance.

The essays in this book are so diverse and some of them are so "meaty", situating fascinating areas of research in this diverse field within wideranging historical and theoretical landscapes, and viewing parts of the field through different lenses to great effect, that it would have benefited from a longer introduction, giving a clearer indication of what the essays within are about, and why these essays in particular were chosen to represent new perspectives and directions in STS. Although it gives a strong take home message, the conclusion could have done more to bring together the essays preceding it. As a result, the reader could be left with an overall impression of STS as a collection of fascinating, but largely siloed topics. However, the book does provide diverse and engaging examples of new perspectives and directions in the field, which is, after all, what it set out to do.

This book is of great interest to students or professional researchers who want to gain a snapshot of some of the many and diverse current themes in STS, the rich history of the ideas behind them, and visions of where they might go next. It may also attract readers with an interest in the complex relationships between science, technology and society, and the myriad of ways in which these relationships are currently studied and understood. 